\title{
EJEMPLOS DE VICARIANZA EN COMUNIDADES DE CRUSTACEOS DE LAGUNAS ESTEPARIAS
}

\author{
Miguel Alonso \\ Dpto. Ecología, Fac. Biología, Universitat de Barecelona. Avenida Diagonal, 645. 08028 Barcelona. \\ Palabras clave: Branchiopoda, Diaptomidae, Communities, Biogeography, Ecology.
}

\author{
ABSTRACT \\ SOME EXAMPLES OF VICARIANCE IN CRUSTACEAN COMMUNITIES OF \\ STEPPIC LAGOONS
}

\begin{abstract}
New information on the historical biogeography and on the phenomena on allopatric speciation and dispersion arises from the study of the crustaceans of the steppic Spanish water bodies. In this paper, the communities of phyllopods and copepods of temporary fresh-watersare analyzed.

The community structure is similar in steppic environments throughout the world and the regularity observed in the association of certain biological types is remarkable. However the specific composition varies considerably on a regional basis when comparing different areas that are very distant, or even in areas that are closely related biogeographically. In most cases, a common ecological meaning is confered to these communities, and thus the different regional manifestations are interesting examples of vicariance.

In the Iberian Peninsula these regional differences are very clear. North to Central System, the communities are characterized by Triops cancriformis simplex, Lepidurus apus and Cyzicus tetracerus. In the south, T. cancriformis mauritanicus and $C^{\prime}$. grubei are characteristic. On the other hand, species found in communities of both areas, as Daphnia similis, reveal some morphological differences which can be interpreted as indices of allopatnc subspeciation.

The fact that there are endemismes, as is the case of Cyzicus grubei, and restricted areas of distribution lead one to question the supposed facility of dispersion, which is typically attributed to these organisms because of their capacity to produce forms of resistance. This also supports the hypotbesis of the considerable age of the freshwater steppic Iberian fauna. Therefore the permanence of these organisms through time could find its explanation in the continuous and conditions from the Tertiary to present time rather than in a greater efficiency in the colonizing strategies.
\end{abstract}

\section{INTRODUCCION}

Las aguas epicontinentales de la región Mediterránea en general y de la Península Ibérica en particular debieron servir de escenario a procesos evolutivos interesantes debido a su situación marginal respecto a las fluctuaciones climáticas que tuvieron lugar desde finales de la Era Terciaria. El área de distribución de una fauna esteparia antigua que debió tener cierta continuidad en el Terciario fue fragmentada finalmente por las últimas glaciacionesa que afectaron desigualmente a los países de la Europa Meridional. Así se generaron distribuciones discontinuas que a grandes rasgos concuerdan con la disyunción iberocáspica pero que en realidad constituyen un rosario de áreas relictas alrededor del Mediterráneo cuyo aislamiento geográfico se ve reforzado por las características climáticas actuales.

Limnética 3: 81-89 (1987)

(C) Asociación Española de Limnologia, Madrid. Spain
Los medios esteparios constituyen así un marco excelente para el estudio de la especiación o subespeciación alopátrica. No todas las especies que han visto repartidas sus poblaciones en núcleos discontinuos se comportan de la misma manera. Los diaptómidos y algunos quidóridos destacan por su capacidad para la especiación rápida (Margalef, 1983; Frey, 1982), por lo que no es extraño que parte de las especies actuales sean postglaciales. En los eufilópodos la situación parece diferente ya que su evolución morfológica ha sido menor; por ejemplo, se conocen restos de Triops del Triásico prácticamente idénticos a los actuales. Sin embargo, esto no implica que su tasa de evolución sea menor o más lenta que la del resto de los grupos. Muchas veces los cambios genotípicos no tienen expresión fenotípica, o ésta no se manifiesta en caracteres evidentes (Simpson, 1953), dando lugar a complejos de especies crípticas. Schopf et al. (1975), 
Zaida

Notostraca

$T$. cancriformis simplex

Lepidurus apus

Conchostraca

Cyzicus tetracerus

Anostraca

Chirocephalus diaphanus

Branchipus schaefferi

\section{Cladocera}

Daphnia (Ct.)similis

Daphnia (Ct.)atkinsoni

Daphnia obtusa

Moina brachiata

Copepoda

Hemidiaptomus (G.) roubaui

Mixodiaptomus incrassatus

Cyclops strenuus

Encyclops serrulatus

Megacyclops viridis

Diacyclops bicuspidatus

Metacyclops minutus
Tollos

Notostraca

$T$. cancriformis mauritanicus

Conchostraca

Cyzicus grubei

Anostraca

Chirocephalus diaphanus

Streptocephalus torvicornis

Linderiella occidentalis

Cladocera

Daphnia (Ct.)similis

Daphnia (Ct.) sp.

Ceriodaphnia quadrangula

Moina brachiata

Dunhevedia crassa

Copepoda

Hemidiaptomus (G.) maroccanus

Diaptomus cyaneus

Mixodiaptomus incrassatus

Metacyclops minutus

Tabla I. Comunidades de crustáceos de la laguna de la Zaida (Zaragoza, mayo de 1982) y de la laguna de los Tollos (Cádiz. marzo de 1978).

Table I. Crustacean communities ofthe Zaida lagoon (Zaragoza, may 1982)and ofthe Tollos lagoon (Cádiz,march, 1978).

utiliza este argumento para desmitificar la imagen de los «fósiles vivientes» (Limulus) y a la vez establece que a medida que se profundiza en la taxonomía de un grupo, su tasa de evolución aumenta.

El resultado es que, a través de diferentes procesos evolutivos e históricos, se ha llegado a distribuciones disjuntas de taxones muy relacionados y de ecología similar, que pueden interpretarse como casos de vicarianza.

El concepto de vicarianza se ha hecho en ocasiones extensivo a las comunidades, en un contexto mucho más general y marginal del estrictamente biogeográfico (Udvardy, 1969). El término comunidad vicaria serviría para definir colectivos de especies diferentes que se desarrollan en ecosistemas similares de regiones distanciadas y tienen una composición de tipos biológicos y estructura trófica equivalente.

El aislamiento de comunidades enteras y su posterior evolución no parece el origen más probable de las comunidades vicarias aunque estaría más de acuerdo con los postulados vicaristas (Croizat et al., 1974). En la mayor parte de los casos se interpreta la evolución de comunidades de estructura similar como un fenómeno de adaptación convergente de las diferentes especies a características ecológicas determinadas (Orians \& Paine, 1983). Comunidades de lagartos y aves de desiertos de diferentes lugares del mundo ofrecerían ejemplos ilustrativos de convergencia (Orians \& Solbrig, 1977; Pianka, 1975).

En este trabajo se pasa revista a posibles casos de vicarianza en algunos de los grupos más representativos de especies esteparias, como notostráceos, anostráceos, concostráceos, cladóceros y diaptómidos. Por otro lado se intenta ampliar el concepto de vicarianza a las comunidades comparando inventarios de diferentes partes de España y analizando la particular distribución de algunas especies.

El material que ha servido de base a los comentarios sobre la biogeografía y la ecología de las especies españolas que se presentan a continuación, procede 
de aproximadamente 200 lagunas esteparias repartidas por todo el país y muestreadas en el periodo que va desde 1976 hasta 1985.

\section{VICARISMO EN BIOTAS ESTEPARIOS}

\section{Notostráceos}

Pertenecen a uno de los grupos de crustáceos más antiguos que se conocen. Se originaron entre el Carbonífero y el Triásico y en la fragmentación de sus poblaciones debió jugar un papel importante la deriva continental. Sólo se conocen dos géneros, Triops y Lepidurus. El primero se encuentra mejor adaptado a ambientes esteparios (Gauthier, 1928) y posee una docena de especies muy próximas morfológicamente y de significado ecológico similar en aguas de todo el mundo (Bayly \& Williams, 1973; Boutin, 1982; Cole, 1968; Rzoska, 1961).

En la región mediterránea aparecen tres especies de Triops. Triops granarzus, de amplia distribución en Africa, Oriente Medio y Asia, se encuentra también en Calabria (Cottarelli \& Mura, 1983), Triops numidicus en el norte de Africa y Triops cancriformis en Europa y el norte de Africa. Ghigi (1921) describe cuatro formas de Triops cancrzformis: mauritanicus, simplex, cancriformis y apulius. Esta última, muy próxima a la simplex, constituiría, según Gauthier (1934) una raza muy localizada. Longhurst (1955) considera razas geográficas a las tres restantes. Triops cancriformis cancriformis se encuentra ampliamente distribuido por Europa y aparece en la Camarga (Aguesse \& Dussart, 1956) y en el norte de Italia (Cottarelli \& Mura, 1983). Triops cancriformis simplex vive en España (al norte del Sistema Central y Levante), Cerdeña (Stella et al., 1972), Argelia y Túnez (Gauthier, 1928) Y Triops cancriformis mauritanicus en España (al sur del Sistema Central y Baleares) y Marruecos (Boutin, 1982). Triops cancriformis simplex y Triops cancriformis mauritanicus constituirían un ejemplo de subespeciación alopátrica de dos formas que actualmente presentan una ecología muy similar pero que mantienen su aislamiento geográfico.

\section{Concostráceos}

El origen de los concostráceos se ha situado en el Devónico inferior y se supone que la distribución de los géneros se debe a la deriva continental (Tasch, 1979). A consecuencia de esto su modelo de distribución corresponde al de un grupo con géneros escasos y cosmopolitas, y especies de distribución restringida.

Se conocen pocas especies en la región mediterránea, pero sin embargo, no faltan los ejemplos de diferenciación regional. En Messina (Sicilia) vive una
Leptestheria de posición sistemática incierta pero muy afín a Leptestheria corticri (Cottarelli \& Mura, 1983) que podría constituir una subespecie o raza geográfica. El género Cyzicus ofrece un ejemplo muy ilustrativo de vicarianza con dos especies morfológicamente muy próximas entre sí, con exclusión geográfica y ecología muy similar. Cyzicus tetracerus se encuentra ampliamente distribuido por la región mediterránea y en España aparece en la cuenca del Ebro, mientras que por su parte, Cyzicus grubei considerada como un endemismo español, habita en la cuenca del Duero y al sur del Sistema Central.

\section{Anostráceos}

Se tienen registros fósiles de anostráceos del Devónico, todos pertenecientes a grupos extinguidos. Dentro de las familias actuales, sólo se conocen fósiles de la de los artémidos, que datan del Pleistoceno (Tasch, 1969).

Los países que se supone han conservado condiciones áridas a través del Pleistoceno (Península ibérica, norte de Africa, Europa Oriental, Asia Menor) poseen, en la actualidad, regularidades faunísticas notables y actualmente podrían constituir focos relictos de distribuciones que fueron mucho más amplias durante el Terciario. En ellos es característica la presencia de muchos géneros y relativamente pocas especies (Alonso, 1985; Gauthier, 1928), pero la fauna de anostráceos es muy uniforme y los ejemplos de diferenciación regional son escasos. Branchinecta cervantesi de España y Branchinecta orientalis de los países al este de Hungría (Brtek, 1959) constituirían el único ejemplo de vicarianza dada su proximidad morfológica y ecológica; sin embargo serían deseables estudios taxonómicos más concluyentes.

En Italia la situación es diferente. Por un lado es más pobre en ambientes esteparios y por otro su fauna sufrió reiteradas extinciones, la última y más drástica durante el Plioceno, que dejó gran parte de la península cubierta por las aguas del Mediterráneo. Actualmente sólo posee tres géneros (la Península Ibérica nueve: Artemia, una especie; Branchinecta, dos especies; Tanymartix, una especie; Branchipus, una especie; Streptocephalus, una especie; Branchinella, una especie; Chirocephalus, una especie; Branchinectella, una especie; Linderiella, una especie), Branchipus, Tanymastix y Chirocephalus, aparte de Artemia (Cotarelli \& Mura, 1983), muy diversificados en ambientes montanos y con poblaciones hasta el momento endémicas de un sólo lago como Branchipus alpinus, Chirocephalus rnarchessonii y Chirocephalus sibyllae. Es cuestionable si el origen de estas especies se produjo por aislamiento geográfico, por ejemplo en cordilleras aisladas durante el Plioceno, o por especiación ecológica tras colonizar ambientes libres de la 


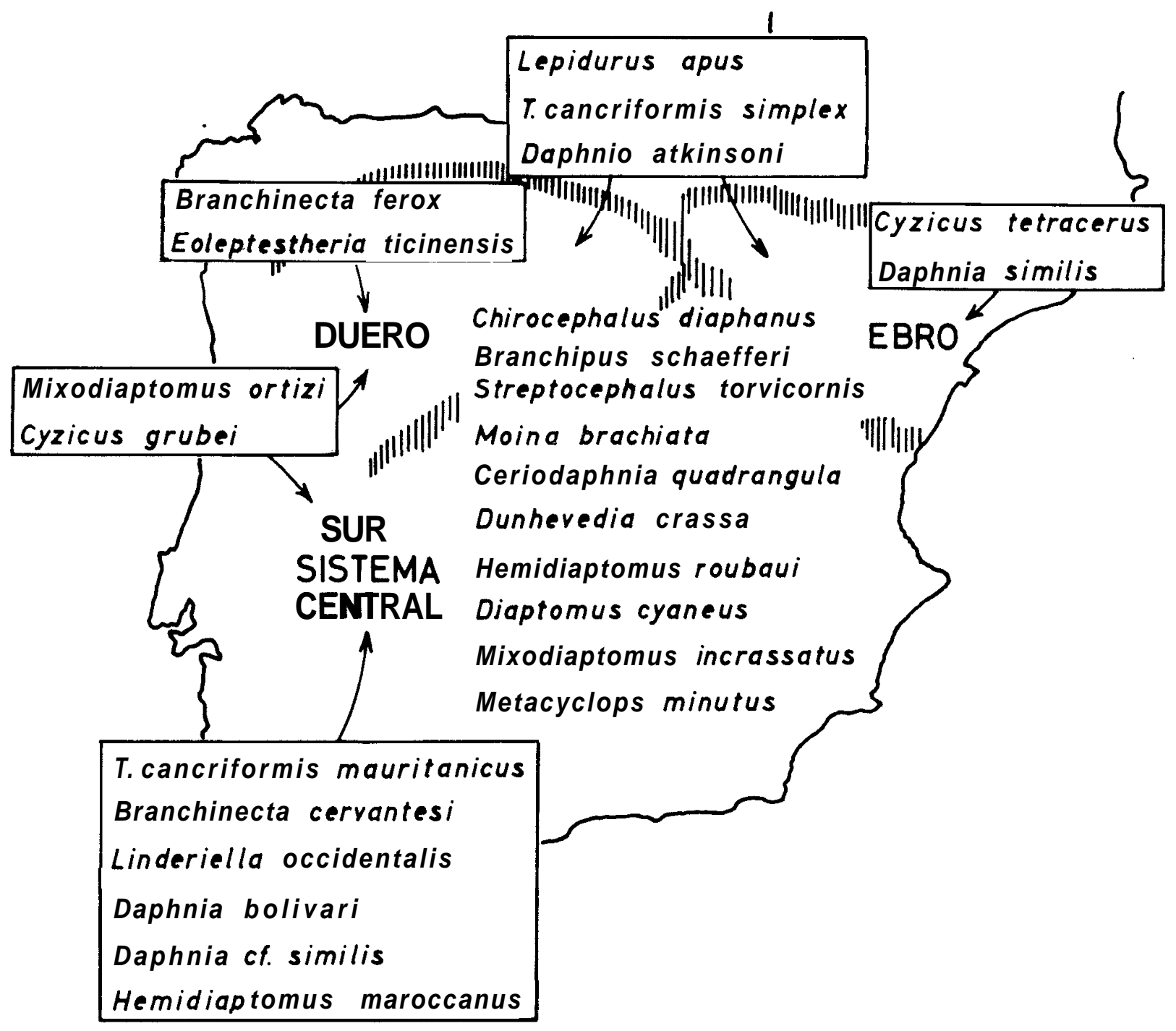

Figura 1.- Distribución en España de las especiesde crustáceos más características de ambientes esteparios. El rayado divide las tres regiones biogeograficas más importantes desde el punto de vista de la distribución de estas especies: Cuenca del Duero, Cuenca del Ebro, y Sur del Sistema Central. En el centro figuran las especies comunes a las tres regiones y en los recuadros las que observan tipos de distribución más o menos restringidos.

Fig. 1.- Spanish distribution of the most characteristic species of steppic environments. The main biogeographical regions from the point of view ofthe distribution ofthese species (Duero basin, Ebro basin and South of the Central System) appear outlined by the striped area. In the center of the map the species common to the three regions are represented, and in the squares, the species with more or less restricted distribution are included.

competencia de otras especies. En cualquier caso constituyen casos muy interesantes de especiación alopátrica.

\section{Cladoceros}

Su dispersión inicial debió ser anterior a la separa- ción de los continentes, como justifica el cosmopolitanismo de la mayoría de los géneros. Posteriormente, su gran capacidad de dispersión y colonización ha conducido a una diversificación notable. Actualmente se conocen aproximadamente 450 especies, muchas de las cuales corresponden en realidad a complejos de especies de gran afinidad taxonómica (Frey, 
1980), cuyo estudio se ha iniciado en algunos casos empleando técnicas de taxonomía morfológica muy detalladas, de electroforesis (Hann, 1980) y de cruzamientos (Shan \& Frey, 1968).

La tendencia de los cladóceros a formar razas locales es muy acusada e incluso pueden aparecer especies diferentes sin procesos de alopatria. Por ejemplo, se conoce la coexistencia de nueve especies de Ephemeroporus en Florida (Frey, 1982b) y diferenciaciones enzimáticas entre especies de Eurycercus del norte y el sur de Norteamérica sin que existan barreras físicas que interrumpan el flujo génico (Hann, 1980). La radiación evolutiva de los cladóceros lleva a tal grado de especialización que permite la coexistencia de especies muy próximas en un mismo lago segregadas por su ecologia, comportamiento o fenología (Frey, 1980)

La distribución de los cladóceros mediterráneos tiene un fondo más ecológico que histórico, y son pocos los casos de alopatria en taxones realmente afines. Dumont et al., (1979), cita dos subespecies de Alona elegans que se excluyen mutuamente en el norte de Africa, la ssp. lebes (occidental) y la ssp. elegans (oriental). Daphnia similis ofrece un ejemplo interesante en España relacionado con la vicarianza. Esta especie posee dos formas cuya distribución sigue el modelo mencionado para Triops y Cyzicus, al norte y al sur del Sistema Central. Las formas del norte, de la cuenca del Ebro, corresponden a la forma típica, mientras que las del sur poseen algunas características, tales como la longitud de la espina, la curvatura del cuerpo y la estructura del yelmo, próximas a la Daphnia carmata o a la $D$. carinuta cf. thomsoni que describe Gauthier $(1928,1929)$ en el norte de Africa o en el Sahara Central. El estudio sistemático aún no ha revelado caracteres que permitan clasificar ambas formas como diferentes. Por el momento, las poblaciones del norte y el sur podrían representar valores extremos dentro del rango de variación morfológica que podrían ser interpretados como un indicio de subespeciación alopátrica.

\section{Diaptómidos}

Los diaptómidos constituyen el grupo de calanoides más extendido en las aguas continentales. Se encuentran entre los organismos más interesantes desde el punto de vista ecológico y biogeográfico. Por otro lado, su elevada tendencia a la especiación, mayor que en otros copépodos, ha favorecido la existencia de gran cantidad de subespecies, variedades y ecotipos de difícil situación taxonómica (Margalef, 1983; Stella, 1979).

Las faunas mundiales se encuentran notablemente diferenciadas, tanto a nivel de continentes (Brehm,
1967) como de pequeñas regiones biogeográficas y la distribución actual es resultado de la acción combinada de factores ecológicos e históricos (Alonso \& Armengol, 1981). Las diferencias en el poblamiento de las tres penínsulas mediterráneas, Ibérica, Itálica y Balcánica, reflejan los sucesivos aislamientos a que se vieron sometidas a través de las fluctuaciones climáticas ocurridas desde principios del Terciario. De manera que en la actualidad la fauna de diaptómidos se encuentra dividida en cuatro bloques de composición especifica muy diferente: Uno en la Mediterránea occidental (Península Ibérica y norte de Africa), otro en la Mediterránea oriental (Península Balcánica y Turquía), otro centroeuropeo, que desciende hacia el sur aprovechando regiones de cierta altura y clima húmedo y temperado, y, el cuarto, el de las boreoalpinas. En la Península Itálica faltan los representantes esteparios, en parte porque el poblamiento es más reciente y en parte por la relativa escasez de zonas áridas.

Los géneros Hemidiaptomus, Diaptomus y Mixodiaptomus constituyen los ejemplos más interesantes de diferenciación regional en aguas esteparias. Hemidiaptomus consta de 15 especies subdivididas en dos subgéneros, Hemidiaptomus s. str. y H. (Gigantodiaptomus) (Kiefer, 1978). Todas ellas se sitúan a lo largo de un gradiente de cambio morfológico en sentido Este-Oeste que va desde la Península Ibérica hasta Asia. En la Mediterránea occidental Gigantodiaptomus está representado por un grupo compacto de especies (roubaui-maroccanus-ingens) taxonómicamente muy próximas y de ecología muy similar. $H$. (G.) roubaui vive en la Península Ibérica, la Camarga y Cerdeña, $H$. (G.) maroccanus en el sur de España y Marruecos y $H$. $(G$.) ingens en Argelia y Túnez. Aunque las dos primeras especies son simpátricas en Andalucía, el complejo roubaui-maroccanus-ingens, podría considerarse constituido por especies vicarias. En Europa Central y Oriental $H$. (G.) amblyodon, tiene una distribución muy amplia y podría haber dado lugar a $H$. (G.) hungaricus, al oeste de los Cárpatos (Hungría, Rumanía). Por su parte, (H. (G.) superbus muestra una curiosa disyunción entre Centroeuropa y el Caspio. El subgénero Hemidiaptomus s. str. falta en el Mediterráneo occidental (su referencia más cercana, H. (H.) ingens, es en Túnez, Gauthier, 1928) y se encuentra extraordinariamente diversificado en el Este de Europa formando un rosario de endemismos que va desde el Caspio hasta los Balcanes.

En el género Diaptomus el gradiente de cambio morfológico es de tipo latitudinal (Pirocchi, 1948), de manera que en Europa hay una sucesión de especies de norte a sur; Diaptomus gracilis sería la forma más septentrional, D. castor, el lusitánico y B. castaneti aparece en los Pirineos y en el Sistema Central francés. Más al sur, y dentro de las especies de distribu- 
ción mediterránea occidental, $D$. cyaneus es el que disfruta de una expansión más amplia. Aparece en ambientes bien diferentes y parece que sus numerosas formas locales no llegan, en muchos casos, a la categoría de subespecie (Kiefer, 1974). En España se conoce el complejo formado por las subespecies cyaneus y admotus (Miracle, 1978), cuya identidad no está clara ya que parece que ambos pueden vivir en ambientes montanos o esteparios indistintamente. Posiblemente estas subespecies no pasen de ecotipos, como parece suceder con las formas major y typica de Diaptomus castaneti o con formas más o menos grandes y pigmentadas con ciertas variaciones alométricas, de Neolovenula alluaudi y Mixodiaptomus incrassatus, adaptadas a la vida en aguas más o menos someras y temporales respectivamente. Diaptomus kenitraensis del Atlas marroquí y D. kenitraensis var. ibérica de España (Dussart, 1964) podrian constituir otro buen ejemplo de vicarianza, aunque para Kiefer (1979) la diferencia entre ambas subespecies no está clara.

Mixodiaptomus laciniatus es una especie boreoalpina. En la región Mediterranea cuenta con dos formas próximas adaptadas a ambientes esteparios: $\mathrm{Mi}$ xodiaptomus laciniatus atlantis y Mixodiaptomus ortizi. La primera vive en el Atlas marroquí y en la Península Ibérica, mientras que la segunda constituye un endemismo español. Ambas especies presentan áreas disjuntas de distribución, $M$. ortizi vive en las regiones esteparias occidentales y M. laciniatus atlantis en las orientales (Alonso, 1984).

\section{VICARISMO EN COMUNIDADES ESTEPARIAS}

Las Comunidades de crustáceos de aguas esteparias proporcionan numerosos ejemplos de convergencia estructural. Desde el punto de vista ecológico todos se caracterizan por la capacidad de soportar grandes fluctuaciones ambientales de tipo aleatorio. Por otra parte, su composición por tipos biológicos se repite en aguas de todo el mundo, siendo la asociación más frecuente, en las más complejas: 1-2 especies de notostráceos, 1-2 especies de concostráceos, hasta 4-5 especies de anostráceos, 3-5 especies de calanoides y varios cladóceros, ciclópidos y ostrácodos (Aguesse \& Dussart, 1956, Boutin, 1982; Gauthier, 1928; Rzoska, 1961; Stella \& Margaritora, 1968; Stella et al., 1972).

La historia evolutiva de cada grupo de los citados, no ha sido equivalente en la constitución de las diferentes manifestaciones regionales de las comunidades. Los más antiguos mantienen sus características biológicas originales independientemente de su tasa de evolución, que en cualquier caso ha sido divergente.
Estos, tras su dispersión podrian haber tenido el papel de «fundadores» de las comunidades de aguas temporales.

En los calanoides, por el contrario, se dan casos muy claros de convergencia evolutiva. En lagunas esteparias de los cinco continentes se han descrito asociaciones de varias especies de calanoides que, al menos desde el punto de vista trófico, parecen tener cierta importancia (Hutchinson, 1967). Las especies de mayor tamaño (5-6 mm) son: en Sudamérica Pseudoboeckella sp. pl., en Australia Boeckella major (Bayly, 1964), en Africa, Europa y Asia Hemidiaptomus sp. pl. y en Norteamérica Diaptomus novemdecimus. Las relaciones filogenéticas entre estas especies son lejanas: las Boeckella son centropágidos, del hemisferio Austral y más antiguas que los diaptómidos, procedentes del norte. Todas constituyen ejemplos de analogía convergente o "pseudovicarianza» (Udvardy, 1969).

Entre las adaptaciones más sobresalientes de estos organismos a las fluctuaciones destacan la resistencia a la sequía mediante la producción de huevos durab le $y$ fases de quiescencia, la resistencia a los cambios osmóticos bruscos (formas eurihalinas, poiquilosmóticas... Beadle, 1943) y la velocidad de los procesos biológicos como crecimiento y alcance de la madurez sexual (Rzoska, 1961).

Dentro de una misma región biogeográfica, las diferencias específicas locales entre comunidades vicarias se acentúan a medida que las comunidades se complican y van introduciéndose especies raras. En la región Mediterranea, la mayoría de comunidades esteparias cuentan con un fondo de especies de distribución más o menos generalizada como Mixodiaptomus incrassatus, Neolovenula alluaudi, Dunhevedia crassa, Moina brachiata, etc. (Alonso \& Comelles, 1984), y son especies pertenecientes al grupo de los notostráceos, anostráceos, concostráceos, diaptómidos y algunos cladóceros las que amplifican las distancias faunísticas.

Una situación ideal de comunidad vicaria sería aquella en que las especies componentes fuesen a su vez también vicarias de las de la otra comunidad homóloga. En España existen algunas aproximaciones de las que se ha tomado la más ilustrativa como ejemplo. En la tabla I figuran los inventarios de las lagunas de la Zaida y de los Tollos, situadas respectivamente en la cuenca del Ebro y del Bajo Guadalquivir. Ambas lagunas son de carácter estepario, temporales, someras, poco mineralizadas y fangosas. Las diferencias específicas más significativas se producen precisamente entre los eufilópodos y diaptómidos que ya constituían casos aislados de especies vicarias al norte v al sur del Sistema Central. Se destacan así en ambas comunidades dos auténticos «colectivos vicarios» formados por Triops cancriformis simplex, $\mathrm{Cy}$ - 
zicus tetracerus, Hemidiuptomus roubaui y Daphnia similis en la Zaida y Triops cancriformis mauritanicus, Cyzicus grubei, Hemidiaptomus maroccanus y Daphnia cf. similis en los Tollos.

Sin embargo, en la mayor parte de los casos los ejemplos de comunidades vicarias tienen relaciones de similaridad más laxas, y su afinidad atañe a tipos biológicos. La diferenciación local de las comunidades tiene su origen en la desigual distribución de las especies, lo que nos llevaría a generalizar sobre las posibles comunidades más frecuentes en las diferentes cuencas españolas. El esquema de la figura 1 ilustra el modelo de distribución de las diferentes especies. Las más cosmopolitas aparecen en las menos complejas como únicos constituyentes. Lepidurus apus, Daphnia atkinsoni y Triops cancriformis simplex caracterizan las comunidades al norte del Sistema Central. Branchinecta ferox y Eoleptestheria ticinensis son exclusivas de la cuenca del Duero mientras que Cyzicus tetracerus y Daphnia similis lo son de la del Ebro. Mixodiaptomus ortizi y Cyzicus grubei siguen una franja occidental que emparenta las estepas del norte y del sur. Por último, Triops cancriformis mauritanicus, Linderiella occidentalis, Daphnia bolivari, Daphnia cf. similis y Hemidiaptomus maroccanus serían los representantes más característicos de las comunidades situadas al sur del Sistema Central.

\section{CONCLUSIONES}

La disposición de las regiones esteparias españolas se presta a fenómenos de aislamiento, relictismo y especiación alopátrica. Respecto a la fauna esteparia de crustáceos, la Península puede dividirse en tres zonas biogeográficas: dos al norte del Sistema Central, bien individualizadas (una en la cuenca del Duero y la otra en la cuenca del Ebro) y una tercera, más amplia que abarca todas las comarcas al sur del Sistema Central.

Las relaciones biogeográficas entre estas tres zonas son en ocasiones más estrechas con otros países del Mediterráneo que entre ellas mismas. El sur de España parece más afín a Marruecos que al resto de los países norteafricanos, que al norte de España y por supuesto que a los países al este de la Península Ibérica. Por su parte las estepas del norte de España guardan sus relaciones faunísticas más afines con el sur de Francia, Cerdeña, Argelia, Túnez, Israel e incluso con países mucho más alejados del este de Europa.

Existe la duda si la fauna esteparia ibérica de aguas epicontinentales es realmente antigua o procede de inmigraciones recientes a causa del incremento de la aridez en los últimos tiempos (Margalef, 1947). Esto constituye un planteamiento muy interesante si se quiere interpretar el origen de las actuales distribucio- nes, de los casos de diferenciación local y de las relaciones biogeográficas entre países. Ambos procesos (inmigración, relictismo) pueden haber actuado de manera combinada hasta llegar a configurar la situación actual. Sin embargo, hay razones para pensar que al menos parte de nuestra fauna es anterior al Pleistoceno. Las diferencias faunísticas entre el norte y el sur de España por un lado, y la similitud de Andalucía con Marruecos por otro, reflejan distribuciones que pudieron tener lugar, ya durante el Mioceno, cuando la Península se encontraba dividida por el estrecho Bético y no existía solución de continuidad entre Gibraltar y el noroeste de Africa. Parece además, que las condiciones áridas no se interrumpieron totalmente durante el Pleistoceno, al menos en las cuencas del Guadalquivir y del Ebro (Plans, 1969), aunque sin embargo sí que pudieron sufrir reducciones lo suficientemente importantes como para aislar poblaciones favoreciendo la aparición de endemismos ( $\mathrm{Cyz} i$ cus grubei, Mixodiaptomus ortizi). Las disyunciones entre la Península Ibérica y el este de Europa, son también una prueba de la antigüedad de la fauna esteparia ibérica. Branchinecta cervantesi, de la Mancha, constituye verosímilmente una reliquia de la distribución mucho más generalizada que pudo tener Branchinecta orientalis durante el Terciario.

Los casos de vicarianza representan adaptaciones funcionales similares en comunidades alopátricas. Los procesos evolutivos que han conducido hacia estas regularidades en aguas esteparias de todo el mundo van implícitos en el propio significado ecológico de las comunidades. Su función más importante es el reciclado de recursos de escaso valor energético (detritus) en las etapas iniciales de la sucesión anual de los ecosistemas acuáticos temporales. Se entiende pues que la escasa especialización trófica de las especies y la pobreza de relaciones interespecíficas no haya dado pie a procesos de evolución. Parece más lógico pensar que la evolución hacia las características comunes de las comunidades vicarias haya sido por convergencia, y no a nivel de comunidades sino de sus especies contituyentes. Buena prueba de ello es que a la uniformidad de tipos biológicos se puede superponer una gran diversidad taxonómica en pares de comunidades análogas.

En la desigual composición específica de las comunidades vicarias intervienen factores ecológicos e históricos. En el primer caso las especies poseen una ecología similar pero una distribución geográfica diferente. En el segundo caso, las especies pueden ser simpátricas pero se diferencian por su desigual tolerancia a parametros ambientales. En ambos casos pueden darse casos de auténtica vicarianza entre especies, sobre todo en áreas geográficas vecinas entre las que la diferenciación genética se mantiene dentro de unos límites (razas, subespecies y ecotipos). 


\section{BIBLIOGRAFIA}

Aguesse, P.C. \& B.H. Dussart, 1956. Sur quelque crustacés de Camargue et leur Ecologie. Vie et Milieu, 7, 4: 481-520.

Alonso, M., 1984. The genus Mixodiaptomus Kiefer, 1932. (Copepoda, Diaptomidae) in Spain. Hydrobiologia 118: 135-146.

Alonso, M., 1985. A survey of the Spanish Euphyllopoda. Misc. Zool., 9: 179-208.

Alonso, M. \& J. Armengol, 1981. Distribució dels Diaptómids Ibéncs en relacio amb árees geográfiques próximes: Facto r histórics i ecológics. Treb. Inst. Cat. Hist. Nat., 9: 135-145.

Alonso, M. \& M. Comelles, 1984. A preliminary grouping of the small epicontinental water bodies in Spain and distribution of Crustacea and Charophyta. Verh. Internat. Verein. Limnol, 22: 1.699-1.703.

Bayly, I.A.E., 1964. A revision of the Australian Species of the freshwater genera Boeckella and Hemiboeckella (Copepoda: Calanoida). Australian J. Marine Freshwater Res., 15: 180-238.

Bayly, I.A.E. \& W.D. Williams, 1973. Inland waters and their ecology. Longman, Victoria (Australia), 314 págs.

Beadle, L.C., 1943. An ecological survey of some inland saline waters of Algeria. J.Linn. Soc. (Zool)., 4 1:218-242.

Boutin, C., 1982. Doneés nouvelles sur la repartition et l'ecologie des Notostracés du Maroc (Crustacés, Branchiopodes). Bull. Fac. Sci. Marrakech, 1, 1:1-7.

Boutin, C., L. Lesne, A. Thiery, 1982. Ecologie et typologie de quelques mare temporaires a isoetes d'une région ande du Maroc occidental. Ecología Mediterránea 8(3): 31-56.

Brehm, V., 1947. Reflexiones sobre relaciones zoogeográficas de la fauna de aguas dulces de la Península Ibérica. Pub. Inst. Biol. Apl., 4: 53-74.

Brtek, J., 1959. Branchinecta ferox (M. Edw.) in der Slowakei und Bemerkungen zu einigen paleartichen Arten der Gattung Branchinecta. Biología, 14(3): 197-2 12

Cole, G.A., 1968. Desert limnology. In: Drsert Biology (Brown, G.W. ed.): 423-486. Academic Press. New York \& London. 635 págs.

Cottarelli, V. \& G. Mura, 1983. Anostraci, Notostraci, Concostraci (Crustacea: Anostraca, Notostraca, Conchostraca). Consiglio Nazionale delle Richerche. Guide per il riconoscimento delle specie animali delle acque interne italiane. 18, 71 pags.

Croizat, L., G.J. Nelson \& D.E. Rosen, 1974. Centers of origin and related concepts. Syst. Zool. 23: 265-287.

Dumont, H.J., P. Laureys \& J. Pensaert. 1979. Anostraca, Conchostraca, Cladocera and Copepoda from Tunisia. $H y$ drobiologia, 66,3: 259-274.

Dussart, B.H. 1964. Copepodes d'Espagne. Bull. Soc. Zool. Fr., 2-3:117-125.

Frey, D.G., 1980. On the plurality of Chydorus sphaericus (O.F. Müller) (Cladocera, Chydoridae) and designation of a neotype from Sjaels, Denmark. Hydrobiologia, 69: 83-123.
Frey, D.G., 1982. Questions concerning cosmopolitanism in Cladocera. Arch. Hydrobiol. 93, 4: 484-502.

Frey, D.G., 1982 b. Relocation of Chydorus barroisi and related species (Cladocera, Chydondae) to a new genus and description of two new species. Hydrobiologia, 86: 231-269.

Gauthier, H., 1928. Recherches sur la faune des raux continentales de l'Algérie et de la Tunisie. Minerva, Algeria, 416 págs.

Gauthier, H., 1929. Cladoceres et Ostracodes du Sahara Central. Bull. Soc. Hist. Nat. Af. N., 20: 143-162.

Gauthier, H., 1934. Contribution a I'etude de l'Apus cancriformis et de ses variations dans l'Afrique du Nord (Euphyllopode Notostracé). Bull. Soc. Sc. Nat. Maroc., 14: 1-15.

Ghigi, A., 1921. Richerche sui Notostraci di Cirenaica e di altri paesi del Mediterraneo. Atti. Soc. Ital. Sci. Nat., 60: 161-188.

Hann, B.J., 1980. Population differentiation in the Eurycercus (Bullatifrons) species complex (Chydoridae, Cladocera) in Eastern North America. Ph. D. Thesis, Indiana Univ. Xl, 165 págs.

Hutchinson, G.E., 1967. Planktonic Copepoda. In: A. Treatise on Limnology (Hutchinson ed.): 624-695. J. Wiley \& Sons. N.Y. and London. 1.115 págs.

Kiefer, F., 1974. Revision der cyaneus -gruppe "der Gattung Diaptomus (Sens. str.) (Crustacea, Copepoda, Calanoida). Zool. Anz. 192: 279-288.

Kiefer, F. 1978. Freilebende Copepoda. In: Das Zooplankton der Binnengewässer, 2, Teil: 1-343. Schwizerbart, Stuttgart. Margalef, R., 1947. Estudios sobre la vida en las aguas continentales de la región endorreica manchega. P. Inst. Biol. Apl., 4: 5-51

Margalef, R., 1983. Limnologia, Omega, Barcelona. 1.010 págs.

Margraf, J. \& B. Maass, 1982. Zur Okologie der Temporären Süsswasserflachseen des Tafelbergs «Giara di Gesturi» auf Sardinien. Spixiana, 5(1): 69-99.

Miracle, M.R., 1978. Composición especifica de las comunidades zooplanctónicas de los Pirineos y su interés biogeográfico. Oecologia aquatica, 3: 167-191.

Orians, G.H. \& R.T. Paine, 1983. Convergent Evolution at the Community Level. In: Coevolution (D.J. Futuyma \& M. Slatkin. eds.): 431-458. Sinauer associates. Sunderland, Massachusetts. 555 pags.

Orians, G.H. \& O.T. Solbrig (eds.), 1977. Convergent Evolution in Warm Deserts. Dowden, Hutchinson \& Ross, Stroudsburg, Pennsylvania.

Pianka, E.R., 1975. Niche relations of desert lizards In: $E c o-$ logy and Evolution of Communities (M.L. Cody \& J.M. Diamond, eds.): 292-314. Harvard University Press, Cambridge, Massachusetts.

Pirocchi, L., 1948. Signification biogeographique de la distribution de quelques espèces de Diaptomides. Verh. Inter. Verein. Limnol., 10: 364-370.

Plans, P., 1969. Problemas del endorreismo español. Rev. Rral Acad. Ciencias Ex. Fis. y Nat., 63: 271-309.

Rzoska, J., 1961. Observations on tropical rainpools and ge- 
neral remarks on temporary waters. Hydrobiologia, 17: 265-286.

Schopf, J.M.T., D.M. Raup, S.J. Gould \& D.S. Simberloff, 1975. Genomic versus morphologic rates of evolution: influence of morphologic complexity. Paleobiology, 1: 63-70.

Shan, R.K. \& Frey, D.G., 1968. Induced interbreding between two stocks of a chydorid cladoceran. BioScience, 18 : 203-205.

Simpson, G.G., 1953. The Major Features of Evolution. Columbia Univ. Press. New York, N.Y., 434 págs.

Stella, E., 1979. Considerazioni biogeografiche sui Diaptomidi (Copepoda, Calanoida) delle acque dolci italiane. Lav Soc. Ital. Biog., 6: 316-328.
Stella, E. \& F. Margaritora, 1968. La fauna ad entomostraci di acque astatiche del Lazio. Rend. Acc. Naz. dei XL, 18: $1-59$.

Stella, E., F.G. Margaritora \& V. Cottarelli, 1972. La fauna ad Entomostraci di acque astatiche della Sardegna nord orientale. Rend. Acc. Naz. 22: 163-210.

Tasch, P. 1969. Branchipoda. In: Treatise on Invertebrate Paleontology (R.C. Moore, ed.): 128-191. Geol. Soc. Am., Boulder, Colorado and the Univ. of Kansas Press, Lawrence.

Tasch, P., 1979. Crustacean branchiopod distribution and Speciation in Mesozoic lakes of the Southem Continents. $A n-$ tarct. Res. Ser. 30:65-74.

Udvardy, M.D.F. 1969. Dynamic Zoogeography, Van Nostrand Reinhold C.O. New York. 444 págs. 